\title{
Generation of confined plasma balls propagating along discharge channels: A comparison with ball lightning
}

\author{
Francis Théberge, ${ }^{*}$ Gaston Nadeau $\odot$, and Vivian Issa \\ Defence Research and Development Canada, Valcartier Research Centre, 2459 De la Bravoure Road, Québec, QC, Canada G3J 1X5 \\ François Vidal $\odot$ and Jean-Claude Kieffer \\ Institut National de la Recherche Scientifique-Énergie Matériaux Télécommunications, 1650 Lionel-Boulet Boulevard, \\ Varennes, QC, Canada J3X $1 S 2$
}

(Received 14 October 2019; revised manuscript received 27 December 2019; accepted 12 February 2020; published 5 March 2020)

\begin{abstract}
Ball lightning is a mysterious phenomenon reported by numerous eyewitnesses. The very limited instrumental characterization of ball lightning recorded in nature has maintained the mystery surrounding this phenomenon and given rise to many theories on their generation mechanism. In this work, we reproduced conditions in the laboratory similar to thunderstorms and observed the generation of luminous plasma balls. The formation of these plasma balls is based on the confinement of the ejected plasma from the ground into the lower pressure channel produced along the path of the discharge. The spectral, temporal, and spatial characterizations done in this work can give some indications on the formation of ball lightning and more particularly its containment with quasiconstant diameter, its average speed, its erratic path, and the various colors of ball lightning observed in nature.
\end{abstract}

DOI: 10.1103/PhysRevResearch.2.013266

\section{INTRODUCTION}

The origin of ball lightning (BL) formation has attracted the attention of scientists for centuries [1-6] and remains one of the most mysterious meteorological phenomena that has not obtained yet a widely accepted scientific explanation. The properties of BL are based mainly on the reports of eyewitnesses and vary appreciably. The instrumental characterizations of BL generated in nature are rare $[7,8]$ and some of those are still questioned [9]. Typically, BL is produced in the vicinity of an active thunderstorm and its formation is generally associated with cloud-to-ground (CG) lightning strike. BL is usually observed close to the ground, with a spherical or ellipsoid shape, moving at a speed of a few meters per second. Its lifetime can range from 1 to $10 \mathrm{~s}$, and the observed colors are various, ranging from white, yellow, red, purple, green, or even changes color during its lifetime.

There are numerous models and experiments proposed to explain and reproduce BL, which can generally be divided into two classes, according to whether the energy source is internal or external. These observations and models are recently reported in Ref. [9] and some of those are briefly reviewed below. The most recent of the internal-energy

\footnotetext{
*francis.theberge@drdc-rddc.gc.ca

Published by the American Physical Society under the terms of the Creative Commons Attribution 4.0 International license. Further distribution of this work must maintain attribution to the author(s) and the published article's title, journal citation, and DOI.
}

theories was proposed by Abrahamson and Dinniss [10] and refined by several groups [7,11-16], suggesting that BL is caused by $\mathrm{CG}$ lightning striking the soil, producing a cloud of filamentary silicon networks whose oxidation in ambient oxygen generates heat and induces a glow. Another model of internal-energy source is based on hydrated plasma where produced ions from discharges are trapped by water molecules in air, which increase the ion fluorescence lifetime up to 0.1 to $1 \mathrm{~s}$, as demonstrated for experiments done in high humidity air environments $[17,18]$. The best-known model for the externalenergy theory was proposed by Kapitza [19], suggesting that an intense radio frequency electromagnetic field could provide the necessary energy to form and sustain BL. Based on this external-energy model, a great number of microwave experiments have been conducted for the generation of fireballs [20-25].

Other experiments have been done with natural thunderclouds and Ref. [7] involved a total of eight artificially triggered lightning strikes using the rocket-and-wire technique. The triggered lightning strikes were directed toward various material samples, and among these tests, some cases produced a glowing ball above metallic plates and had a total duration of $\sim 300$ ms. Recently in Ref. [8], the observation of possible BL produced after a CG lightning strike was recorded using high-speed cameras, providing the first potential results on their spectral characteristics. The BL colors recorded in the visible spectrum indicated that most of the emission lines are radiated by neutral silicon, iron, and calcium. These elements are the main components of soil; it was therefore inferred that the observed BL was generated by the CG lightning striking the soil on the ground. 
BL generated in nature is regarded as a rare phenomenon; but this belief might be based on erroneous reasoning. In Ref. [26], Smirnov compiled more than 2600 cases of BL observation, and it has been estimated that the probability for the observation of BL over the lifetime of a person is low, around $0.2 \%$. However, with these statistics and considering the distribution of these cases according to distance from the observer, it has been approximated that the frequency density [27] of BL occurrence is $W_{\mathrm{BL}} \approx 10^{1.3 \pm 1.0} \mathrm{~km}^{-2} \mathrm{yr}^{-1}$. This density is of similar order than the frequency density of ordinary lightning appearance [28]. Therefore, associating BL with the occurrence of a lightning strike does not make it a rare phenomenon. However, since BL is usually observed at close range, near the ground, and given that $\mathrm{BL}$ is produced around the impact zone of a lightning strike, the probability of observing lightning strike at such proximity and in such conditions is also low, although the probability of its occurrence is not.

In our work, we reproduced conditions similar to those observed in a thunderstorm for the electric field at ground level, and we observed confined plasma ball (PB) for almost all electric discharges performed. The color and spectral composition of the PB were directly associated with the chemical composition of the ground electrode where the discharge strikes. As detailed in this work, the luminous intensity and lifetime of observed PB are also dependent on ground chemical composition and the amplitude of the electric field around the electrode. The propagation path, speed, spectral signature, and spherical/ellipsoid shape of the PB were similar with BL's eyewitness reports and were linked to the advection of the ground material injected into the lower air pressure channel produced along the discharge path.

\section{EXPERIMENTAL SETUP}

To generate BL, a lightning discharge must be initiated which generally involves two major processes: the downward moving negative leader and the upward moving return stroke, the first one preceding the second [29]. To reproduce similar conditions in the laboratory, we used a hybrid high-voltage source consisting of a Tesla coil connected in series with a high-load 30-kV capacitance [30]. The experimental setups used for this work are detailed in Appendix A. The Tesla coil was used to mimic the generation of a lightning leader and the lower voltage of the high-load capacitance simulated the return stroke with an ambient electric field of 15 to $60 \mathrm{kV} / \mathrm{m}$, which is much lower than the breakdown voltage of $3 \mathrm{MV} / \mathrm{m}$ in ambient air, but similar to the electric field at ground level during a thunderstorm [31]. Table I summarizes the discharge parameters used in our tests [30] and compares them with thunderstorm/lightning conditions taken from Refs. [6,29]. Evidently, the voltage used in our smaller-scale experiment is lower, but the resulting ambient electric field and the air density of the heated channel are similar. The diameter of the heated air channel in our work is smaller than what is observed in nature, given the lower current density achieved in the laboratory (two to eight times lower); this also explains the smaller diameter of the PB produced in our experiments as compared to the BL's diameter reported by eyewitnesses. The second impact of the smaller discharge channel diameter is the faster decrease of its excitation temperature [32]. For
TABLE I. Comparison of the discharge parameters of the experimental setup with those of lightning during a thunderstorm.

\begin{tabular}{lcc}
\hline \hline Parameters & Laboratory & Lightning \\
\hline Electric field $(\mathrm{kV} / \mathrm{m})$ & $15-60$ & $5-15$ \\
Peak current density $\left(\mathrm{kA} / \mathrm{cm}^{2}\right)$ & $0.2-0.4$ & $0.7-3$ \\
Heated channel diameter $(\mathrm{mm})$ & $5-20$ & $10-300$ \\
Channel air density $\left(\mu \mathrm{g} / \mathrm{cm}^{3}\right)$ & $\sim 10$ & $10-100$ \\
\hline \hline
\end{tabular}

larger lightning channel produced, the excitation temperature can remain above $4000 \mathrm{~K}$ up to $1 \mathrm{~s}$ [32], which could explain also the longer lifetime of BL produced in nature. Another constraint by performing this experiment in dry air environment (humidity ranging from $30 \%$ to $45 \%$ for avoiding water condensation on high voltage sources) is the limited formation of hydrated plasma $[17,18]$. Such effect can be observed when the water concentration in air is sufficiently high to trap a large proportion of produced ions, delaying their recombination and increasing their fluorescence lifetime up to $1 \mathrm{~s}$.

Subsequently, another series of tests using a long and thin plasma channel produced by the filamentation of ultrashort laser pulses [30] was performed in the laboratory-scale experiment. During the spectral characterization, these plasma channels allowed to guide the discharge leader precisely along the laser path and to impose a linear propagation on the generated PB.

\section{RESULTS AND DISCUSSIONS}

Figures 1(a)-1(d) present successive frames of a video, taken by a high-speed camera, of a discharge induced by the high-voltage source (without laser guiding). Similar to a discharge from lightning, the development of the air discharge is initiated by the formation of a corona, followed by its selfconstriction into multiple streamers, and the most conductive streamer becomes the leader. The leader, once initiated from the highly stressed electrode, progresses in the gap in successive sequences of corona and streamer formation. The leader's head projects the electrical field from the electrode further into the gap along an erratic path that depends on the presence of initial impurities and their distribution in the air gap. The electric field projected from the leader can be sufficiently high to initiate a leader from the ground electrode, as shown in Fig. 1(a). The progression mechanisms of both positive and negative leaders are analogous in air [33,34]. Once the positive leader meets the negative leader in the air gap, the electrons at the negative leader head recombine with the positive ions at the positive leader head and produce a brighter channel at their intersection [see the green dashed ellipse in Fig. 1(b)], which is often erroneously associated with BL generation. For this experimental setup, the plasma produced along the leader path recombines and heats the air to a temperature of $\sim 4800 \mathrm{~K}$, inducing rapid hydrodynamic expansion. Once the heated air expansion has sufficiently reduced the air density in the channel, a discharge along the leader channel begins to drive the current from the high-load capacitance [see Figs. 1(c) and 1(d)]. During this discharge, the heated air channel expands further, with a radial speed 
(a)

(a)

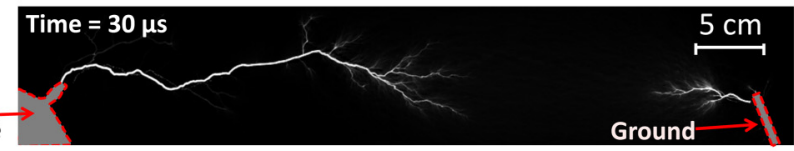

(b)

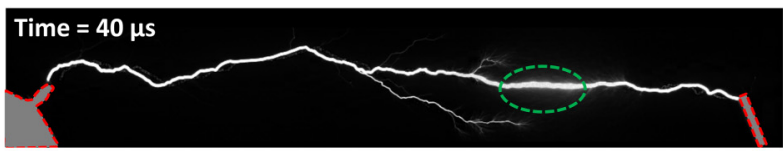

(c)

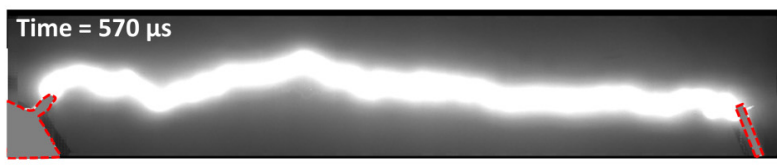

(d)

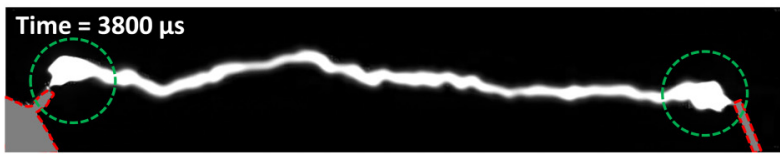

(e)
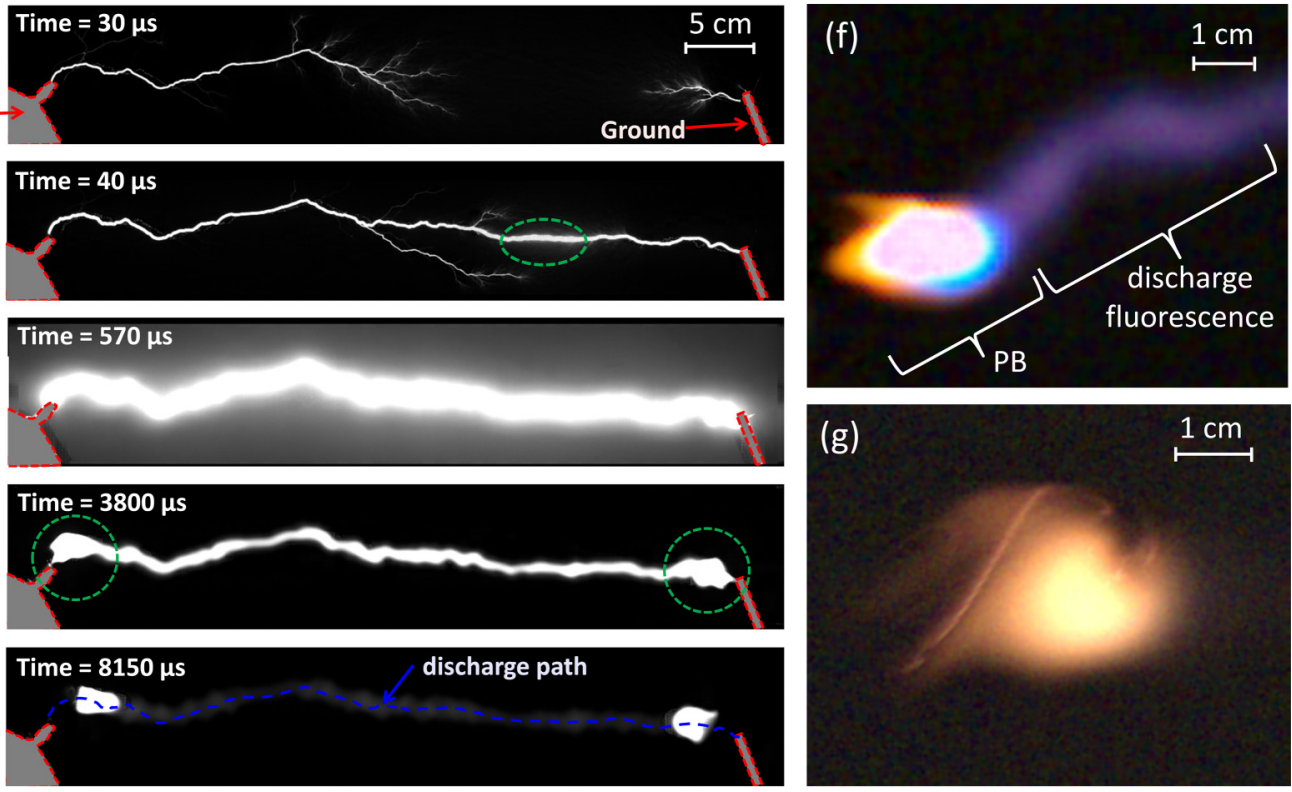

FIG. 1. Successive video frames of discharge over a 50-cm air gap. The video frames, taken with a monochrome high-speed camera, show: (a) the progression of leaders; (b) the connection of leaders (see the green dashed ellipse); (c) the discharge at the peak current; (d) the end of discharge and initiation of PB (see the green dashed circles); and (e) the progression of PB along the initial discharge path (represented by the blue dashed line). Magnified photos taken by a color high-speed camera of PB produced from an iron electrode at (f) the end of the discharge (time $=4.5 \mathrm{~ms}$ ) and $(\mathrm{g})$ the end of the PB's lifetime (time $=17 \mathrm{~ms}$ ). In (f), the white area at the center of the PB corresponds to saturated pixels.

around $5 \mathrm{~m} / \mathrm{s}$ ( $\sim 70$ times slower than the speed of sound), indicating a quasi-equilibrium between the pressure inside the discharge channel and the surrounding ambient pressure. At the end of the discharge [Fig. 1(d)], the temperature of the heated channel is $4800 \pm 500 \mathrm{~K}$ and the air density is reduced.

As shown in Fig. 1(d) at both extremities of the discharge (see green dashed circles), a cloud of plasma is ejected at the point where the discharge connects with the electrodes. At the end of the discharge, the pressure inside the discharge channel becomes lower than the ambient pressure after $\sim 0.5 \mathrm{~ms}$. As a consequence, the ambient air enters this low-pressure channel by its extremities and pushes the cloud of plasma ejected from the electrodes into the initial discharge channel [see Figs. 1(d) and $1(\mathrm{e})$ ]. The result of this mechanism is observed indirectly in the magnified picture of PB shown in Fig. 1(f), where the cloud of plasma formed at the left electrode is pushed by the ambient air entering the channel, which also causes the PB to sweep the remaining plasma ahead of it in the discharge channel [mauve channel in Fig. 1(f)].

During its entire lifetime, the PB is guided along the lowdensity air channel and follows exactly the initial discharge path. As shown in Fig. 1(g) for a magnified picture of the $\mathrm{PB}$ at the end of its lifetime, and for the particular case of iron electrode presented in this image, the iron plasma cloud ejected from the electrode is oxidized by the molecular oxygen from the ambient air entering the channel. Consequently, the PB emission becomes completely orange at the end of its lifetime as a result of fluorescence from excited $\mathrm{FeO}$ molecules. All of the above mechanisms are detailed in the following sections through experimental and theoretical investigations, and PB produced from various electrode ma- terials are characterized in the spectral, temporal and spatial domains.

\section{A. Theoretical models}

The temporal evolution of the current $I$ flowing in the discharge channel is governed by the well-known underdamped resistive-capacitive-inductive equation $I(t)=$ $I_{0} e^{-t R_{\text {air }} / 2 L_{2}} \sin (\omega t)$, shown in Fig. 2(a). In this expression, $t$ is the time, $R_{\text {air }}$ is the resistance of the air channel, $I_{0}$ is the initial current amplitude, $L_{2}$ and $C_{3}$ are the inductance and capacitance of the high-voltage source, respectively, and $\omega=\sqrt{\left(L_{2} C_{3}\right)^{-1}-R_{\text {air }}^{2} /\left(2 L_{2}\right)^{2}}$. The black curve in Fig. 2(b) represents the measured temporal evolution of plasma fluorescence strength in the discharge for a $50-\mathrm{cm}$ air gap when a constant voltage of $+30 \mathrm{kV}$ was applied to the high-load capacitance $C_{3}$ (see Appendix A). As shown in Figs. 2(a) and 2(b), the current flowing in the discharge depends mainly on the inductance and capacitance of the high-voltage source used, and the resulting high impedance of this discharging circuit takes some time to build up the high-current, making the discharge fluorescence to peak around $400 \mu$ s after the leader. This peak of fluorescence corresponds to a peak current of around $80 \mathrm{~A}$ to $100 \mathrm{~A}$, depending on the ambient electric field.

Figure 2(c) shows a simulation of the pressure at the center of the discharge channel as a function of time by using a Lagrangian hydrodynamic model with azimuthal symmetry of the discharge channel. The heating of the channel was modelled by delivering around the center of the channel the underdamped oscillating current shown in Fig. 2(a). The amplitude and range of the power density pulse, which is proportional to the square of the current $I(t)$, were set to 

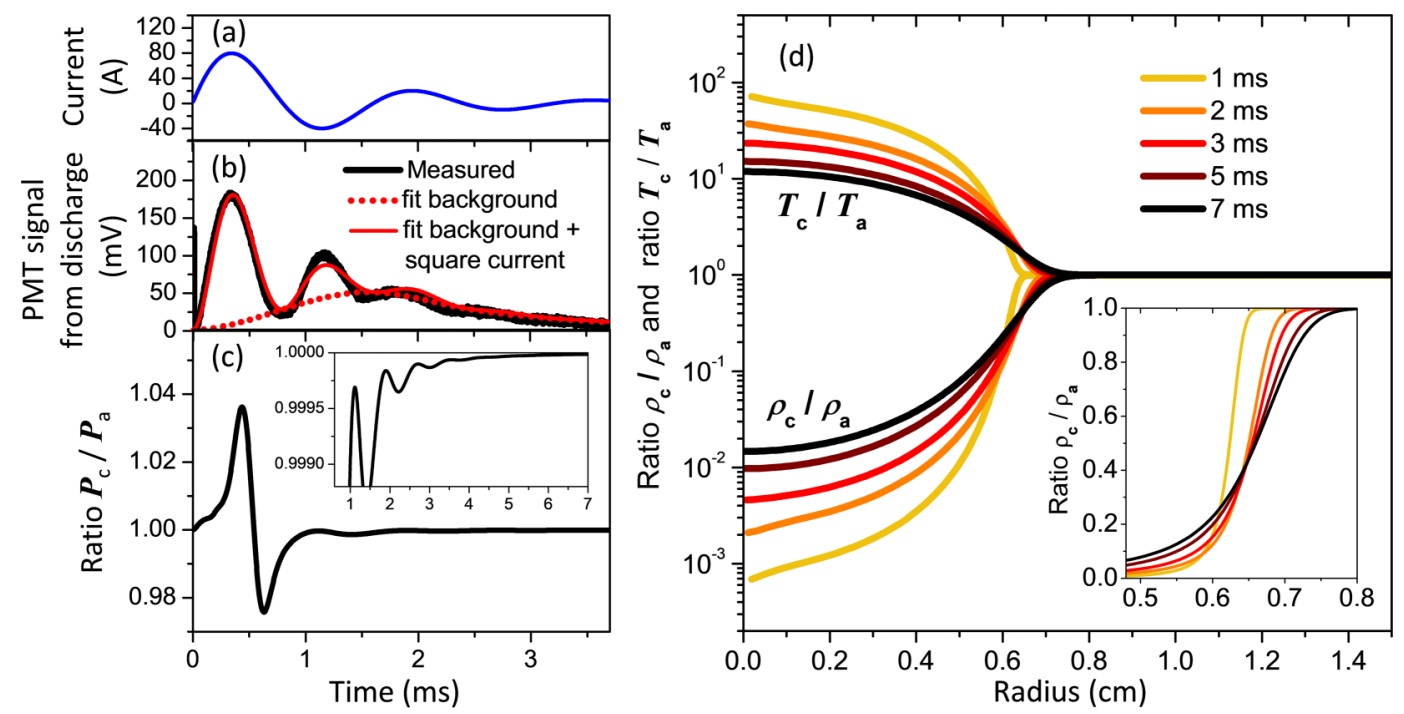

FIG. 2. (a) Current in the discharge calculated by considering $R_{\text {air }}=100 \mathrm{Ohm}$. (b) Measured temporal evolution of the discharge emission (black line) detected by a photomultiplier tube (PMT), and fitted signal with the calculated square current (red line) plus a background emission (red dotted line). (c) Simulated pressure normalized over the ambient pressure at the center of the channel as a function of time. Inset: zoomed ratio of pressure for $t>1 \mathrm{~ms}$. (d) Simulated ratio of the channel air density $\left(\rho_{c}\right)$ over the ambient air density $\left(\rho_{a}\right)$ and ratio of the channel temperature $\left(T_{c}\right)$ over the ambient temperature $\left(T_{a}\right)$ as a function of the radius for different delays after the ignition of the discharge. Inset: zoomed ratio of the air density on a linear scale.

reproduce the observed values for the temperature ( $\sim 4800 \mathrm{~K})$ and diameter of the channel $(\sim 1 \mathrm{~cm})$ after a few milliseconds of evolution. Details are provided in the Appendix B. This simulation indicates that the rapid expansion of the heated air by the power density pulse induces an initial increase of pressure at the center [see Fig. 2(c)], but thereafter, the recoil of the expanding heated air and the extinction of the power density pulse reduce the pressure inside the channel below the ambient pressure. Because of the damped oscillating current source, the pressure inside the channel oscillates as well, but remains below the ambient pressure for time $>0.5 \mathrm{~ms}$. Figure 2(d) shows the simulated radial distribution of the temperature and air density during and after the discharge. It can be observed that the heated channel cools down after the first current peak and only expands slightly with time.

\section{B. Characterization of produced PB}

Figure 3(a) presents a sequence of synchronized video images (color and 2D schlieren) zoomed around the ground electrode, where the evolution of discharge and the formation of PB can be observed. The dark contours in the 2D schlieren images correspond to the strong density gradient at the edge of the discharge channel [see inset in Fig. 2(d) for comparison]. Following $0.5 \mathrm{~ms}$ after the ignition of the leader, the pressure inside the discharge channel is reduced due to the recoil from the radial expansion of the pressure wave and, as shown in Fig. 3(a), the material ejected from the ground electrode is pushed into the discharge channel by the ambient air entering the lower pressure channel by its extremities. The laminar air flow pushing the PB can be observed in Fig. 3(a) for time $\geqslant 4 \mathrm{~ms}$ by the dark stripes behind the $\mathrm{PB}$ and at the center of the channel in the 2D schlieren images. As the $\mathrm{PB}$ progresses into the lower pressure channel, its diameter expands initially through a mechanism similar to a blast wave [35], i.e., the radius expands with time to the power of $2 / 5$ [see Fig. 3(b)]. However, after $\sim 1 \mathrm{~ms}$, the PB's diameter ceased its expansion and remained quasi-constant thereafter because of the containment provided by the lower pressure in the channel and by the abrupt increase in air density at the channel's edge, as shown previously in Figs. 2(c) and 2(d).

Eyewitnesses often report that the BL luminosity is similar to a $100 \mathrm{~W}$ incandescent lightbulb (which corresponds to 5 to $10 \mathrm{~W}$ of light power in the visible), the BL moves at speed around $4 \mathrm{~m} / \mathrm{s}$ [1-6,26,27], and Cen et al. in Ref. [8] observed that the BL luminosity was affected by the ambient electric field. In Figs. 3(c) and 3(d), these parameters have been quantified for the PB produced in the laboratory and correspond very well to the above observations for BL. As shown in Fig. 3(c), the average speed of produced PB corresponds to $3-6 \mathrm{~m} / \mathrm{s}$ and tends to slowly decrease as a function of time. By considering the speed of the air entering the low-pressure channel as a laminar flow and by neglecting the gravitational effect, we obtain the speed of the air entering the channel using the Darcy-Weisbach equation. Consequently, the speed of the PB pushed by this air $\left(V_{\mathrm{PB}}\right)$ is given by $V_{\mathrm{PB}}=\sqrt{D_{c}\left(P_{a}^{2}-P_{c}^{2}\right) / f L \rho_{a} P_{a}}$, where $D_{c}$ is the diameter of the channel (taken from 2D schileren measurement), $P_{a}$ is the ambient pressure, $P_{c}$ is the pressure inside the channel [given by the simulation in Fig. 2(c)], $L$ is the PB propagation length into the channel, $\rho_{a}$ is the ambient air density, and $f=64 \mu_{a} /\left(\rho_{a} D_{c} V_{\mathrm{PB}}\right)$ is the friction factor for laminar air flow where $\mu_{a}=17 \mathrm{~Pa} \cdot \mu \mathrm{s}$ is the dynamic viscosity of ambient air. The calculated value of $V_{\mathrm{PB}}$ is shown in Fig. 3(c) by the red line, and it is in very good agreement with the experimental measurements. For time below $2 \mathrm{~ms}$, it was not possible to calculate $V_{\mathrm{PB}}$ precisely because the electrode shadow partially masked the initial input diameter of the low-pressure channel $\left(D_{c}\right)$ imaged with the schlieren camera. The oscillations in the 

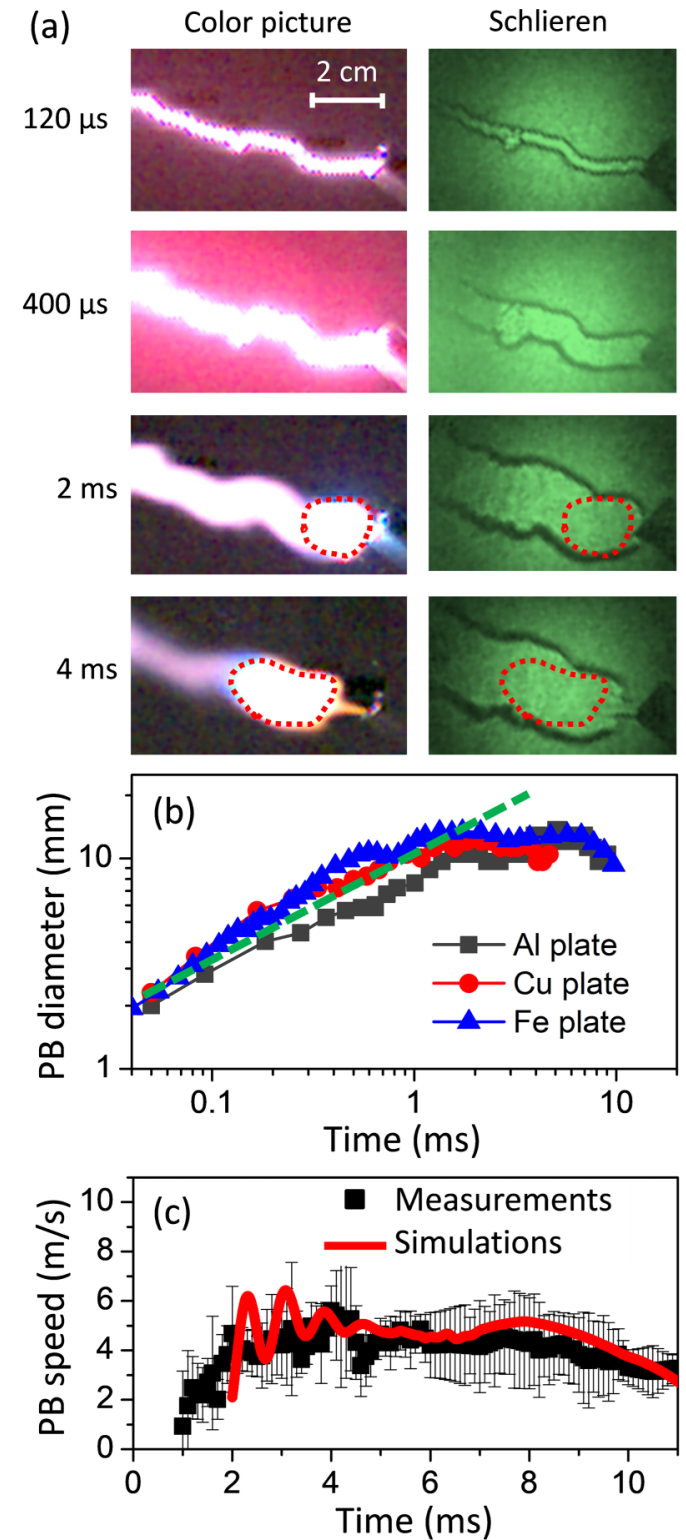
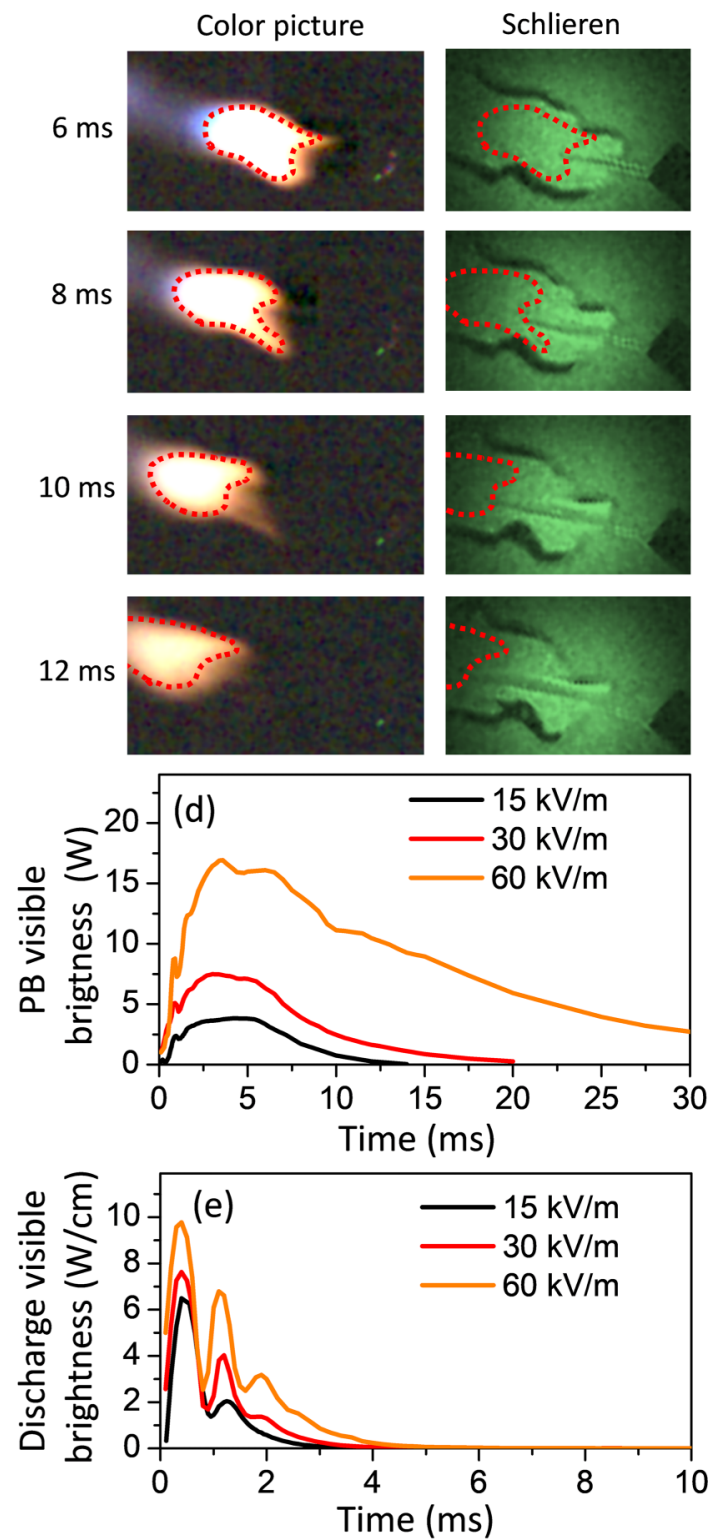

FIG. 3. (a) Synchronized real-color side-images (first and third columns) and 2D schlieren images (second and fourth columns) of discharge and PB formation. The periphery of the PB is highlighted by a red dashed line in both the color and 2D schlieren pictures. (b) PB diameter measured as a function of time for different ground materials. The green dashed line corresponds to 2/5 power law fit. (c) Measured average PB speed and its standard deviation (black squares with error bars) for various experimental configurations. The calculated PB speed (red line) is based on the Darcy-Weisbach equation. (d) Measured average PB brightness in the visible band and (e) linear discharge brightness for different ambient electric fields by using iron electrodes.

value of $V_{\mathrm{PB}}$ mainly observed between 2 and $4 \mathrm{~ms}$ in Fig. 3(c) originate from the oscillating pressure at the channel center [see inset of Fig. 2(c)].

The PB brightness shown in Fig. 3(d), which varies between 3 and $15 \mathrm{~W}$ in the visible band, depends not only on time, but also on the ambient electric field around the electrodes. In our laboratory tests, the PB lifetime according to the decrease of its luminosity at $e^{-1}$ was $9 \mathrm{~ms}$ for an ambient electric field of $15 \mathrm{kV} / \mathrm{m}$; it increased to $20 \mathrm{~ms}$ when the electric field augmented to $60 \mathrm{kV} / \mathrm{m}$. For reference, Fig. 3(e) presents the discharge channel's visible luminosity per unit length (Watt per discharge length) as a function of time, and this luminosity was almost proportional to the square of the current flowing through it. From Fig. 3(e), it can be seen that the brightness of the PB is not related in time to the discharge channel brightness [note the different timescales in Figs. 3(d) and 3(e)]. The PB emission peaks after the discharge and slowly decays with time, and its luminosity is clearly dependent on the ambient electric field, as observed in nature in Ref. [8].

\section{Spectral signature of PB}

To further point out the validity of our experimental setup to reproduce conditions similar to lightning strikes for the formation of BL, we performed a vertical discharge 


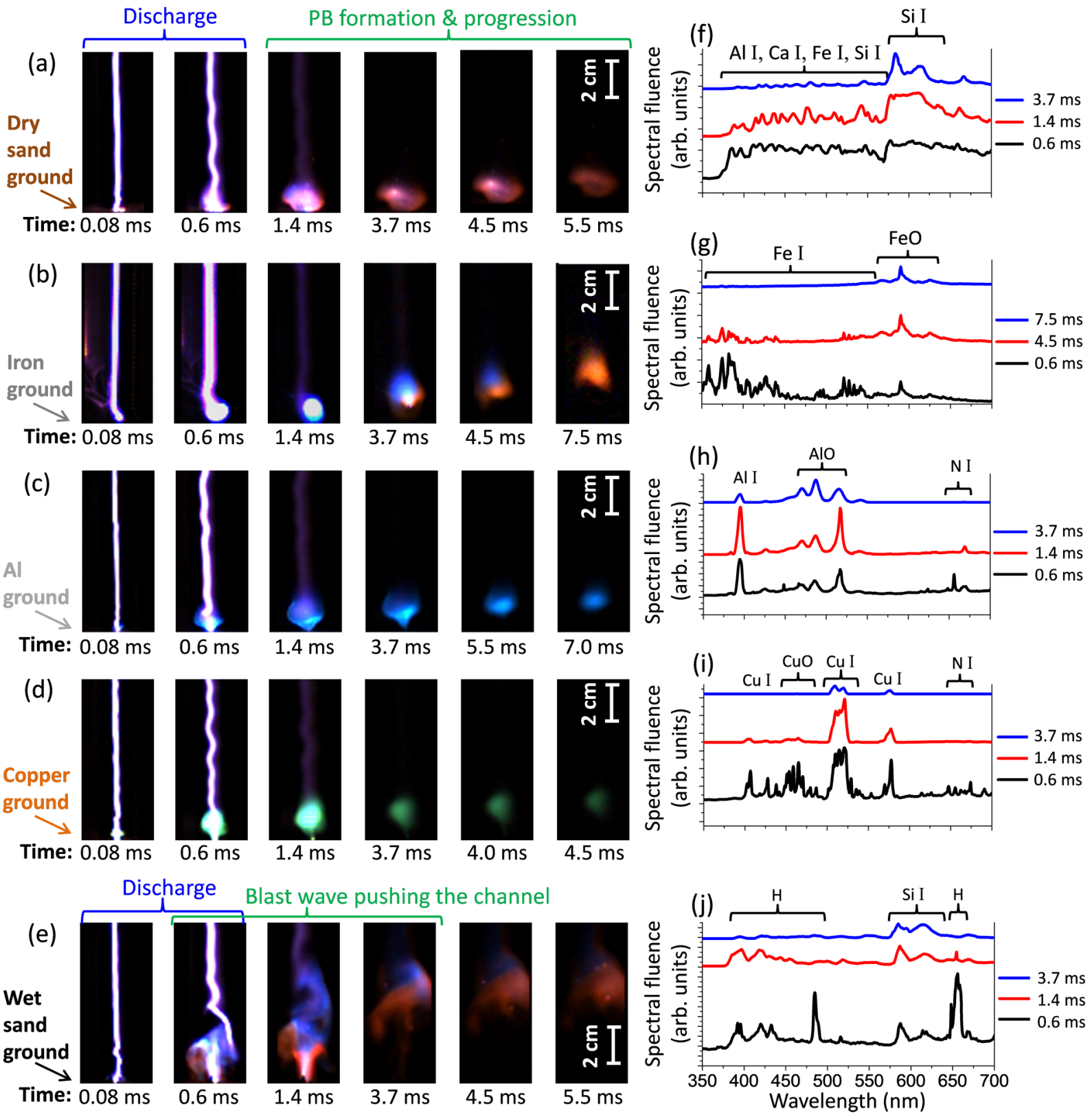

FIG. 4. Successive video frames of $+30 \mathrm{kV}$ DC vertical discharges over a 50-cm air gap taken from the side with a high-speed color camera for a ground plate made of (a) dry sand, (b) iron, (c) aluminum, (d) copper, and (e) wet sand. Spectral emission of the PB at different times for (f) dry sand ground, (g) iron ground, (h) aluminum ground, and (i) copper ground (Note: spectra are vertically shifted for clarity). (j) Spectral emission of material ejected from the wet sand ground.

experiment (HV source on top, ground at the bottom) in which the discharge struck on different ground materials (iron, copper, aluminum, wet sand, and dry sand, shown in Fig. 4). The PB spectra produced above the dry sand ground are very similar to the spectral signature observed in nature [8]. It is also interesting to note that the color evolution of the PB was also very similar to that reported for BL in Ref. [8], but over a shorter timescale, as it was white-purple during most of its lifetime (because of the fluorescence of $\mathrm{Al} \mathrm{I}, \mathrm{Ca}$ I, Fe $\mathrm{I}$, and $\mathrm{Si} \mathrm{I}$ ), changing to red (mainly the fluorescence of $\mathrm{Si}$ I between 570 and $650 \mathrm{~nm}$ ) at the dissipating stage. Other reports of eyewitnesses indicate that $\mathrm{BL}$ can change color during their propagation. Such behavior is observed when the discharge strikes an iron plate at the ground [see Fig. 4(b)]. At the beginning of the PB formation, it is mainly the Fe I fluorescence that is observed, giving a bluish color to the PB. As the PB is pushed into the vertical discharge channel, the upper part of the PB enters the low-air density channel while the bottom part of the PB is pushed by the air at ambient density which contains a much higher oxygen density that will further oxidize the bottom part of the iron PB. Such a predominant oxidation process at the back of the PB generates iron oxide, with the characteristic orange fluorescence from $\mathrm{FeO}$ [see picture at $4.5 \mathrm{~ms}$ in Fig. 4(b)]. As the iron PB progress further into the initial discharge channel, the iron oxidation continues and the PB changes from its initial bluish 
(a)

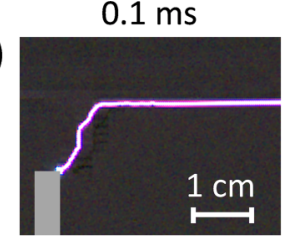

(b)

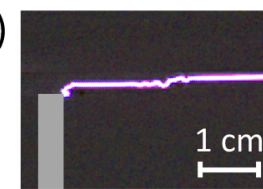

$1 \mathrm{~ms}$
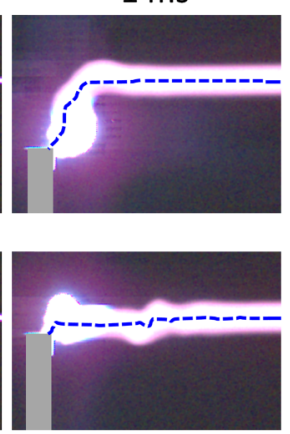

$3 \mathrm{~ms}$
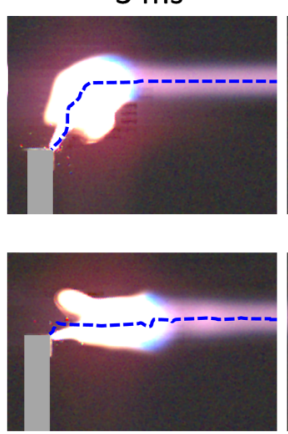

$6 \mathrm{~ms}$
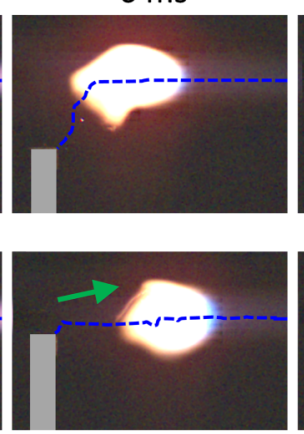

$10 \mathrm{~ms}$
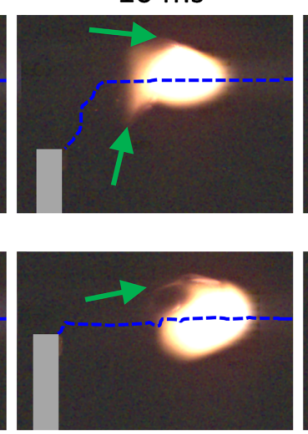

$15 \mathrm{~ms}$
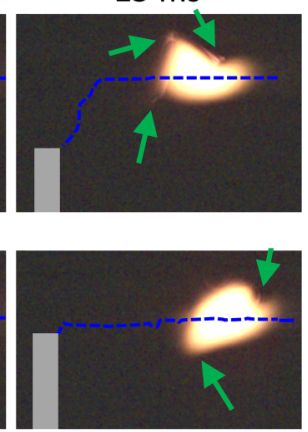

FIG. 5. Successive video frames of $+30 \mathrm{kV}$ discharges. Pictures are taken from the side with a high-speed color camera and for an iron rod ground (represented by the grey zone). Discharges are (a) partially and (b) completely laser-guided along a horizontal path with the plasma filament produced by an ultrashort laser pulse. Blue dashed lines represent the initial discharge paths and green arrows point out vorticity structures developed around the PB.

color to completely orange by the end of its lifetime [see Figs. 4(b) and 4(g)].

Other colors of PB can be generated depending on the ground material on which the discharge strikes. For an aluminum ground plate, the $\mathrm{PB}$ fluorescence is bluish and it is due primarily to $\mathrm{Al} \mathrm{I}$ and $\mathrm{AlO}$, which fluoresce mainly between 380 and $530 \mathrm{~nm}$ [see Figs. 4(c) and 4(h)]. Similarly, for a copper ground plate, the PB remains greenish during its whole lifetime because of $\mathrm{Cu} \mathrm{I}$ and $\mathrm{CuO}$ fluorescence lines are concentrated between 450 and $580 \mathrm{~nm}$, as shown in Figs. 4(d) and 4(i).

Similarly to triggered lightning tests for BL generation in Ref. [7], the formation of PB was not observed for some ground materials, such as wet sand or wood, in our laboratory conditions. In the case of wet sand, the vaporization of water at the discharge strike point produced a strong blast wave that blew out the low-pressure channel produced by the discharge, thus preventing the confinement of the plasma cloud ejected from the ground and the formation of $\mathrm{PB}$, as shown for timing of 0.6 and $1.4 \mathrm{~ms}$ in Fig. 4(e). The cloud of plasma ejected from the wet sand ground is constituted primarily of Si I and hydrogen, as shown by the spectroscopic analysis in Fig. 4(j). However, since the strong blast wave produced by the vaporization of water at the discharge strike pushed away the low-pressure channel, the cloud of materials ejected from the wet sand ground are not confined and therefore expand more. As shown in Fig. 4(e) for timing larger than $1.4 \mathrm{~ms}$, the cloud of ejected material expands over a diameter 2 to 3 larger than the discharge channel and remained at a static height of $4 \mathrm{~cm}$ above the ground surface. Similarly, for the wood ground, the explosion that took place at the discharge strike point on the wood blew out the low-pressure channel produced along the discharge path. These observations support the importance of the radial pressure and density distributions produced by the discharge channel on the formation and the propagation path of $\mathrm{PB}$.

\section{Controlling the path of PB}

To further support the importance of the discharge channel on the formation and displacement of $\mathrm{PB}$, and to clearly show that air convection (upward motion of hot gases) was not a dominant process, Fig. 5 presents the formation of PB and their paths for mainly horizontal discharges. The path of the discharge leader was controlled by a laser beam partly [Fig. 5(a)] or completely [Fig. 5(b)] over its whole horizontal path. The blue dashed lines in Fig. 5 indicate the path of the discharge recorded at $0.1 \mathrm{~ms}$ after the ignition of the leader. For both partly and completely laser-guided discharges, the plasma cloud ejected from the electrode at the discharge strike point is pushed along the discharge channel by the ambient air entering its extremities. In the initial stage of PB formation [see Figs. 5(a) and 5(b) at times of 1 and $3 \mathrm{~ms}$ ], the material ejected at the discharge strike point on the ground electrode is projected at high speed and most of it is injected into the discharge channel.

After the first current peak, the heated channel cools down and the ambient air entering the channel induces advection by pushing the ejected plasma from the ground electrode into the channel, forcing this ejected material to follow exactly the initial discharge path because of the lower pressure in the channel [see Fig. 2(c)] and the abrupt increase of air density at its radial edges [see Fig. 2(d)]. Finally, depending of obstacles around the ground discharge strike point or bifurcations into the discharge path, for examples, the air entering the channel can develop asymmetric velocity distribution across the channel section; which makes twirl the PB inside the channel and eventually induces vorticity structures as pointed out by green arrows in Fig. 5 for time $\geqslant 6 \mathrm{~ms}$.

\section{CONCLUSIONS}

Our experimental results demonstrated the generation of confined PB propagating along discharge channels. The properties of these $\mathrm{PB}$ are in quantitative agreement with many BL's parameters reported by eyewitnesses, namely: (1) the speed, (2) the erratic path, (3) the spectral signature, (4) the brightness, (5) the various colors observed, (6) the stable diameter, and (7) the observation of vorticity structures. The many different models and experiments proposed to explain and reproduce BL can generally be divided into two classes, according to whether the energy source is internal or external. Our work does not discriminate one of them but instead suggests that both classes account for the BL luminosity. 
As clearly presented in Figs. 1(g) and 4(b) for the PB originating from the iron ground plate, the exothermic reaction of iron and oxygen in the PB contributes to lengthening its luminosity, consistently with the Abrahamson and Dinniss [10,36] oxidation model. However, as shown in Fig. 3(d), the external electric field seems to increase the PB's luminosity and its lifetime, and such dependence was also observed in Ref. [8] for natural BL. However, we cannot confirm whether the increase in the PB lifetime and luminosity with the ambient electric field was caused directly by an electroluminescence effect [37], indirectly caused by an increase of the ejected material at the discharge strike point [38] and/or in the generation of a lower-density discharge channel [29] which increased the PB fluorescence lifetime by reducing the collision frequency. Nevertheless, a high-frequency electromagnetic field (radio frequencies $>100 \mathrm{MHz}$ ) was not detected from beginning to end of the PB's lifetime [30,39], indicating that microwave or radio frequencies do not contribute to the formation and confinement of PB. In fact, PB's spherical/ellipsoid form is due to the containment of the plasma cloud ejected from the discharge strike point by the radial pressure and density distribution produced by the discharge channel.

PB speed is determined mainly by the diameter and the pressure inside the discharge channel, and if the air entering the channel develops asymmetric velocity distribution across the channel section, the produced PB begins to twirl inside the channel and eventually develops vorticity structures. The erratic path of $\mathrm{PB}$ is determined by the discharge path itself and, in nature, by the combined effect of wind distribution, which moves and further distorts the lower-pressure channel produced. Previous works and our work have shed considerable light on BL generation mechanisms, however further exploration is required, particularly into the optimal conditions for producing them. Finally, the higher current density in lightning [32] and the contribution of high air humidity producing hydrated plasma $[17,18]$ should be studied to quantify their impacts on the longer emission lifetime of $\mathrm{BL}$ observed in nature.

\section{ACKNOWLEDGMENTS}

This work was supported by a Defence Research and Development Canada Program. F.V. and J.C.K. acknowledge support from the Natural Sciences and Engineering Research Council of Canada. The authors acknowledge technical support from Danny Fortin, Nancy Bérubé, and Pascal Duchesne.

\section{APPENDIX A: EXPERIMENTAL METHODS}

\section{Hybrid high-voltage source and schlieren imaging}

The hybrid AC-DC high-voltage source used in this work consists of a solid-state Tesla coil combined in series with high-load $30 \mathrm{kV}$ capacitance (see Fig. 6). The primary circuit has a large capacitance $\left(C_{1}=470 \mathrm{nF}\right)$ and a small inductance $\left(L_{1}=0.69 \mathrm{mH}\right)$, while the secondary circuit has a large inductance $\left(L_{2}=58 \mathrm{mH}\right)$ and a top load toroid with a small capacitance $\left(C_{2}=141 \mathrm{pF}\right)$. It is important to note that the resistance of the top load toroid $C_{2}$ is practically zero. The primary circuit also includes a triggered high-voltage transistor to synchronize the primary circuit (jitter $<100 \mathrm{~ns}$ )

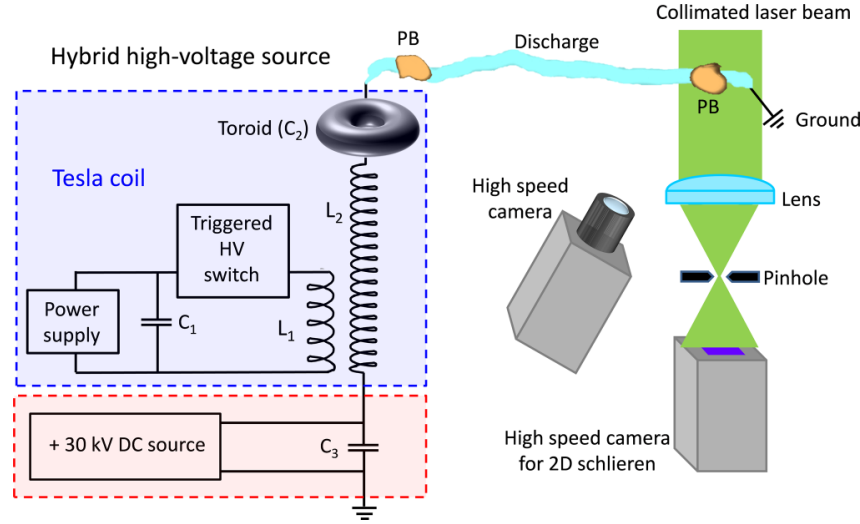

FIG. 6. The schematic of the experimental setup for monitoring the discharges and PB produced from the hybrid AC-DC highvoltage source. The AC high-voltage source is the Tesla coil in the blue-dashed box and the DC high-voltage source is connected to the high load capacitance $C_{3}$ in the red-dashed box. Produced PB was monitored using two synchronized high-speed cameras, one for color imaging and the other for 2D schlieren.

with the high-speed camera or with an ultrashort laser pulse. By inductive coupling, the primary circuit will generate an oscillating current in the secondary circuit (frequency of $\left.55.6 \mathrm{kHz}=\left(\frac{1}{2 \pi}\right) \sqrt{C_{2} L_{2}}\right)$ reaching a peak voltage of $500 \mathrm{kV}$ on the top load toroid, corresponding to a maximal stored energy of $1 \mathrm{~J}$. A 25-mm long metal rod (5-mm diameter) was fixed to the Tesla coil top load toroid to provide a break-out point for the discharges. The high load capacitance $C_{3}$ shown in Fig. 6 can be charged up to $+30 \mathrm{kV}$ by a DC high-voltage power supply, enabling the storage of up to $500 \mathrm{~J}$ of electric energy in this capacitance.

Produced PB was monitored with two synchronized highspeed cameras at a frame rate of $50 \mathrm{kHz}$ and an integration time of $10 \mu \mathrm{s}$. In this setup (see Fig. 6), one camera was used for color imaging and the other for 2D schlieren imaging by using a 1-mm-diameter pinhole [40]. The laser source used for the 2D schlieren imaging was a collimated laser beam with a diameter of $10 \mathrm{~cm}$ (full width at $1 / e^{2}$ ) and emitting a continuous-wave laser with a central wavelength of $532 \mathrm{~nm}$ and an average power of $100 \mathrm{~mW}$.

\section{Laser-guiding of discharge and slitless spectrograph}

The slitless spectrograph was based on a high-speed camera with a 300 lines $/ \mathrm{mm}$ transmission grating positioned $5 \mathrm{~cm}$ in front of the camera imaging lens [8]. This setup shown in Fig. 7 covered a spectral range from 350 to $700 \mathrm{~nm}$ and enabled a spectral resolution of $0.8 \mathrm{~nm} /$ pixel. To maintain a constant wavelength calibration of the spectrograph's field of view, the leader path of the vertical discharge was stabilized by the weak plasma channel initially generated by filamentation of an ultrashort laser pulse (laser beam aligned downward) $[30,39]$. The initial weak ionization of air by the laser beam enables to guide the discharge but it does not affect the final temperature and air density of the heated air channel produced by the discharge because the laser energy absorbed is less than $0.01 \%$ of the electric energy absorbed in air. The laser guiding of electric discharge [30] was done 


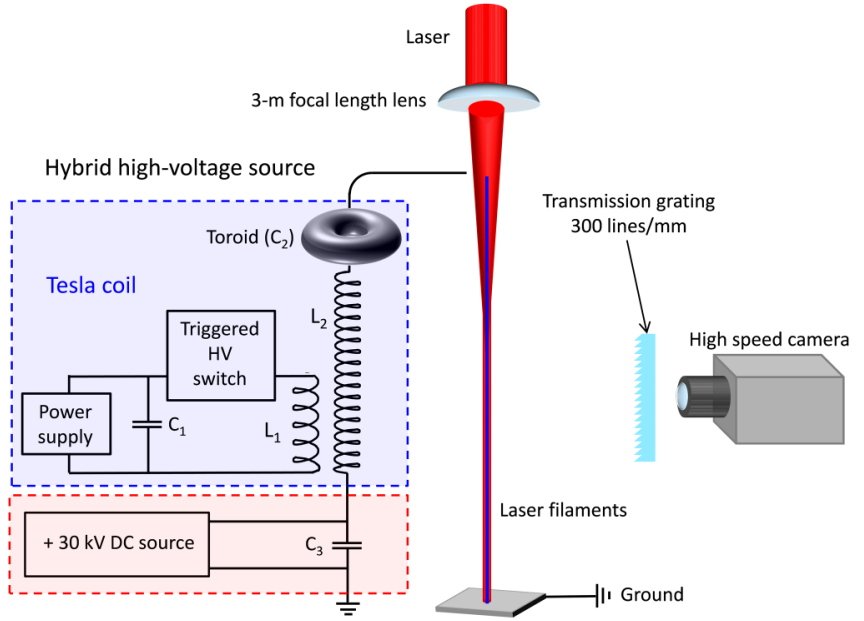

FIG. 7. The schematic of the experimental setup for laserguiding of discharge and the spectroscopic analysis of produced PB using a slitless spectrograph.

with ultrashort laser pulses of $0.15 \mathrm{~J}$ generated from a mobile Ti:Sapphire-based Chirped Pulse Amplification (CPA) system at a repetition rate of $10 \mathrm{~Hz}$ [41]. The laser pulses were negatively chirped to a pulsewidth of $1.02 \mathrm{ps}$ (full width at $1 / e^{2}$ ) and focused using a 3-m focal length lens to produce a plasma filament bundle of a length of $1.5 \mathrm{~m}$ in air. Plasma filaments produced by the ultrashort laser pulses were generated $1 \mathrm{~cm}$ next to the high-voltage electrode. The plasma filament bundle was positioned so that it started slightly before the Tesla coil electrode and the strongest part of the filament bundle produced around the lens focus was positioned above the ground plate. The ultrashort laser pulse was triggered 190 $\mu \mathrm{s}$ after the high-voltage switch of the Tesla coil to match the laser-generation of plasma filaments with one peak of the Tesla coil electric field. During all measurements, laser filaments and the surrounding laser beam did not impact the electrodes.

\section{APPENDIX B: HYDRODYNAMIC LAGRANGIAN MODEL}

In the case of large capacitance and inductance, as in our high-voltage circuit, the plasma fluorescence strength in the discharge is nearly proportional to the square of the current flowing in the channel [42], and $R_{\text {air }}$ remains mainly constant in time [43]. The value for $R_{\text {air }}$ during the discharge was adjusted to $100 \mathrm{Ohm}$ in the calculation to obtain the best fit for the temporal evolution of the measured fluorescence strength [see Fig. 2(b)]. A background signal from both the continuum emission due to radiative recombination [44] and the blackbody emission of the heated air [30] was added to obtain better agreement between the measurement and the calculated square current. This background emission was retrieved from the minima (at times $0,0.8,1.53,2.26$, and $2.98 \mathrm{~ms}$ ) observed in the measured discharge emission [black curve in Fig. 2(b)].

To simulate the evolution of the air radial velocity $u$, mass density $\rho$, temperature $T$ and pressure $p$ of the discharge, we solved the Euler equations,

$$
\rho \frac{d u}{d t}=-\nabla p
$$

together with the equation for temperature (or energy),

$$
C_{V} \frac{d T}{d t}+B_{T} \frac{d \rho}{d t}-\frac{p}{\rho^{2}} \frac{d \rho}{d t}=\frac{1}{\rho}(\nabla \cdot k \nabla T+P),
$$

in cylindrical geometry. In these expressions, $k$ is the heat conduction of air, and $C_{V}=(\partial U / \partial T)_{\rho}$ and $B_{T}=(\partial U / \partial \rho)_{T}$ are the specific heat at constant volume and constant temperature, respectively. It is important to note that the external forces (gravity and friction) were neglected, but a real gas law for air [45] was used in this simulation instead of the perfect gas law. According to Ref. [45], the air pressure can be approximated as

$$
p(\rho, T)=\left(\gamma_{\mathrm{eff}}-1\right) \rho U(\rho, T),
$$

where $\gamma_{\text {eff }}$ is the effective adiabatic index of air, and the internal energy per unit mass as

$$
U(\rho, T)=8.3 \frac{e}{m_{\text {air }}}\left(\frac{T}{10^{4}}\right)^{1.5}\left(\frac{\rho_{a}}{\rho}\right)^{0.12},
$$

where $e$ is the elementary charge and $m_{\text {air }}$ is the mass of an air molecule. The power per unit volume injected in the discharge channel was assumed to be of the form

$$
P(r, t)=P_{0}\left(\frac{I(t)}{I_{0}}\right)^{2} \exp \left(\frac{r^{2}}{r_{0}^{2}}\right),
$$

where $I(t)$ is given by the RLC equation given in the Theoretical models section. For the simulations performed in this work, we used $P_{0}=6 \times 10^{10} \mathrm{Wm}^{-3}$ and $r_{0}=3 \times 10^{-4} \mathrm{~m}$, which corresponds approximately to the radius of the laser-ionized channel [30]. These parameters produced the observed discharge radius and temperature after few milliseconds.
[1] S. Singer, The Nature of Ball Lightning (Springer Science \& Business Media, Berlin, 2012).

[2] J. Barry, Ball Lightning and Bead Lightning (Plenum Press, Berlin, 1980).

[3] D. Turner, Ball lightning and other meteorological phenomena, Phys. Rep. 293, 2 (1998).

[4] M. Stenhoff, Ball Lightning: Unsolved Problem in Atmospheric Physics (Kluwer Academic and Plenum Publishers, Berlin, 1999).
[5] J. Abrahamson, A. V. Bychkov, and V. Bychkov, Recently reported sightings of ball lightning: Observations collected by correspondence and russian and ukrainian sightings, Philos. Trans. R. Soc. London A 360, 11 (2002).

[6] V. Rakov and M. Uman, Lightning: Physics and Effects (Cambridge University Press, Cambridge, 2006).

[7] J. D. Hill, M. A. Uman, M. Stapleton, D. M. Jordan, A. M. Chebaro, and C. J. Biagi, Attempts to create ball lightning with triggered lightning, J. Atmos. Sol. Terr. Phys. 72, 913 (2010). 
[8] J. Cen, P. Yuan, and S. Xue, Observation of the Optical and Spectral Characteristics of Ball Lightning, Phys. Rev. Lett. 112, 035001 (2014).

[9] M. L. Shmatov and K. D. Stephan, Advances in ball lightning research, J. Atmos. Sol. Terr. Phys. 195, 105115 (2019).

[10] J. Abrahamson and J. Dinniss, Ball lightning caused by oxidation of nanoparticle networks from normal lightning strikes on soil, Nature 403, 519 (2000).

[11] G. S. Paiva, A. C. Pavão, E. Alpes de Vasconcelos, O. Mendes, Jr., and E. da Silva, Jr., Production of Ball-Lightning-Like Luminous Balls by Electrical Discharges in Silicon, Phys. Rev. Lett. 98, 048501 (2007).

[12] K. D. Stephan and N. Massey, Burning molten metallic spheres: One class of ball lightning? J. Atmos. Sol. Terr. Phys. 70, 1589 (2008).

[13] T. Ito, T. Tamura, M. A. Cappelli, and S. Hamaguchi, Structure of laboratory ball lightning, Phys. Rev. E 80, 067401 (2009).

[14] G. S. Paiva, J. V. Ferreira, C. C. Bastos, M. V. Dos Santos, and A. C. Pavao, Energy density calculations for ball-lightning-like luminous silicon balls, Phys. Usp. 53, 209 (2010).

[15] T. Ito and M. A. Cappelli, Nanoparticle production in arc generated fireballs of granular silicon powder, AIP Adv. 2, 012126 (2012).

[16] C. L. Porter, G. P. Miley, D. J. Griffiths, and E. Sánchez, Charge on luminous bodies resembling natural ball lightning produced via electrical arcs through lump silicon, Phys. Rev. E 90, 063102 (2014).

[17] A. I. Egorov, S. I. Stepanov, and G. D. Shabanov, Laboratory demonstration of ball lightning, Phys. Usp. 47, 99 (2004).

[18] A. Egorov and S. Stepanov, Properties of short-living ball lightning produced in the laboratory, Tech. Phys. 53, 688 (2008).

[19] P. L. Kapitza, Free plasma filament in a high frequency field at high pressure, Zh. Eksp. Teor. Fiz. 57, 1801 (1969) [Sov. Phys. JETP 30, 973 (1970)].

[20] Y. Ohtsuki and H. Ofuruton, Plasma fireballs formed by microwave interference in air, Nature 350, 139 (1991).

[21] H. Ofuruton, N. Kondo, M. Kamogawa, M. Aoki, and Y.-H. Ohtsuki, Experimental conditions for ball lightning creation by using air gap discharge embedded in a microwave field, J. Geophys. Res. 106, 12367 (2001).

[22] K. D. Stephan, Microwave generation of stable atmosphericpressure fireballs in air, Phys. Rev. E 74, 055401(R) (2006).

[23] J. B. A. Mitchell, J. L. LeGarrec, M. Sztucki, T. Narayanan, V. Dikhtyar, and E. Jerby, Evidence for Nanoparticles in Microwave-Generated Fireballs Observed by Synchrotron X-Ray Scattering, Phys. Rev. Lett. 100, 065001 (2008).

[24] V. Dikhtyar and E. Jerby, Fireball Ejection from a Molten Hot Spot to Air by Localized Microwaves, Phys. Rev. Lett. 96, 045002 (2006).

[25] H.-C. Wu, Relativistic-microwave theory of ball lightning, Sci. Rep. 6, 28263 (2016).

[26] B. Smirnov, The properties and the nature of ball lightning, Phys. Rep. 152, 177 (1987).

[27] B. M. Smirnov, Reviews of topical problems: Physics of ball lightning, Sov. Phys. Usp. 33, 261 (1990).

[28] H. J. Christian, R. J. Blakeslee, D. J. Boccippio, W. L. Boeck, D. E. Buechler, K. T. Driscoll, S. J. Goodman, J. M. Hall, W. J.
Koshak, D. M. Mach, and F. Stewart, Global frequency and distribution of lightning as observed from space by the optical transient detector, J. Geophys. Res. 108(D1), 4005 (2003).

[29] J.-F. Ripoll, J. Zinn, C. A. Jeffery, and P. L. Colestock, On the dynamics of hot air plasmas related to lightning discharges: 1. Gas dynamics, J. Geophys. Res. Atmos. 119, 9196 (2014).

[30] F. Théberge, J.-F. Daigle, J.-C. Kieffer, F. Vidal, and M. Châteauneuf, Laser-guided energetic discharges over large air gaps by electric-field enhanced plasma filaments, Sci. Rep. 7, 40063 (2017).

[31] C. J. Biagi, M. A. Uman, J. Gopalakrishnan, J. Hill, V. A. Rakov, T. Ngin, and D. M. Jordan, Determination of the electric field intensity and space charge density versus height prior to triggered lightning, J. Geophys. Res. Atmos. 116, D15201 (2011).

[32] J.-F. Ripoll, J. Zinn, C. A. Jeffery, and P. L. Colestock, On the dynamics of hot air plasmas related to lightning discharges: 2. Electrodynamics, J. Geophys. Res. Atmos. 119, 9218 (2014).

[33] F. Rizk and G. Trinh, High Voltage Engineering (CRC Press, Boca Raton, 2014).

[34] Y. Raizer and J. Allen, Gas Discharge Physics (Springer, Berlin, 1997).

[35] G. Taylor, The formation of a blast wave by a very intense explosion. I. Theoretical discussion, Proc. R. Soc. London A 201, 159 (1950).

[36] J. Abrahamson, Ball lightning from atmospheric discharges via metal nanosphere oxidation: From soils, wood or metals, Philos. Trans. R. Soc. London A 360, 61 (2002).

[37] J. Liu and X.-C. Zhang, Terahertz-Radiation-Enhanced Emission of Fluorescence from Gas Plasma, Phys. Rev. Lett. 103, 235002 (2009).

[38] D. M. Friday, P. B. Broughton, T. A. Lee, G. A. Schutz, J. N. Betz, and C. M. Lindsay, Further insight into the nature of balllightning-like atmospheric pressure plasmoids, J. Phys. Chem. 117, 9931 (2013).

[39] F. Théberge, J.-F. Gravel, J.-C. Kieffer, F. Vidal, and M. Châteauneuf, Broadband and long lifetime plasma-antenna in air initiated by laser-guided discharge, Appl. Phys. Lett. 111, 073501 (2017).

[40] G. Settles, Schlieren and Shadowgraph Techniques-Visualizing Phenomena in Transparent Media (Springer-Verlag GmbH, Berlin, Germany, 2001).

[41] M. Châteauneuf and J. Dubois, Canadian defense agency acquires new portable terawatt laser, SPIE Newsroom 10, 0506 (2006).

[42] T. Walker and H. Christian, Novel observations in lightning spectroscopy, in Proceedings of the 15th International Conference on Atmospheric Electricity (ICAE) (XV International Conference on Atmospheric Electricity, Norman, Oklahoma, USA, 2014).

[43] P. Castera and P.-Q. Elias, Resistance models applied to the return stroke phase of negative pulsed surface discharges in air, IEEE Trans. Plas. Sci. 42, 1922 (2014).

[44] M. Uman, Lightning (Dover Publications, Mineola, NY, 2011).

[45] B. Zeldovich and Y. Raizer, Physics of Shock Waves and Hightemperature Hydrodynamic Phenomena (Dover Publications, Mineola, NY, 2002). 